\section{Asymmetric cortical and nuclear localizations of WRM-1/ $\beta$-catenin during asymmetric cell division in C. elegans}

\author{
Hisako Takeshita ${ }^{1}$ and Hitoshi Sawa ${ }^{1,2,3}$ \\ ${ }^{1}$ Laboratory for Cell Fate Decision, Riken, Center for \\ Developmental Biology, Kobe 650-0047, Japan; ${ }^{2}$ Division of \\ Bioinformation, Department of Biosystems Science, Graduate \\ School of Science and Technology, Kobe University, \\ Kobe 650-0017, Japan
}

$\beta-C a t e n i n$ can promote adhesion at the cell cortex and mediate Wnt signaling in the nucleus. We show that, in Caenorhabditis elegans, both WRM-1/ $\beta$-catenin and LIT-1 kinase localize to the anterior cell cortex during asymmetric cell division but to the nucleus of the posterior daughter afterward. Both the cortical and nuclear localizations are regulated by Wnts and are apparently coupled. We also found that the daughters show different nuclear export rates for LIT-1. Our results indicate that Wnt signals release cortical WRM-1 from the posterior cortex to generate cortical asymmetry that may control WRM-1 asymmetric nuclear localization by regulating cell polarity.

Supplemental material is available at htttp://www.genesdev.org.

Received April 11, 2005; revised version accepted June 3, 2005.

Asymmetric cell division is a fundamental mechanism for producing cellular diversity (Horvitz and Herskowitz 1992). Asymmetric division often involves the polarized localization and segregation of cell-fate determinants. In Drosophila, the Numb and Prospero proteins are cellfate determinants that are asymmetrically segregated into one of the daughter cells (ganglion mother cell [GMC]) (Betschinger and Knoblich 2004; Roegiers and Jan 2004). During neuroblast divisions, these proteins localize to the basal side of the cell cortex where they are segregated into the GMC. The asymmetric localizations of these molecules are regulated by Bazooka/Par-3, Par-6, and $\mathrm{aPKC}$, which localize to the opposite side, i.e., the apical cell cortex. Similarly, in the Caenorhabditis elegans embryo at the one-cell stage, PAR-3, PAR-6, and aPKC localize to the anterior cortex (Kemphues 2000). Although the mechanisms are not clearly understood, these molecules control the localization of fate determinants (Numb and Prospero in Drosophila and P granules in C. elegans) to the opposite side of the cells through the regulation of cell polarity. Therefore, the cortical local-

[Keywords: Wnt; $\beta$-catenin; WRM-1; cell polarity; asymmetric cell division; C. elegans]

${ }^{3}$ Corresponding author.

E-MAIL sawa@cdb.riken.jp; FAX 81-78-306-3200.

Article and publication are at http://www.genesdev.org/cgi/doi/10.1101/ gad. 1322805 ization of polarity regulators appears to play important roles in asymmetric cell division, although this has not been clearly demonstrated.

After the four-cell stage of C. elegans development, the polarity of many cells, including the EMS blastomere and the T hypodermal cell, is regulated by the Wnt signaling pathway (Thorpe et al. 2000; Korswagen 2002). Unlike the tissue-polarity Wnt pathway, which regulates cell polarity in Drosophila and mammals independent of $\beta$-catenin (Adler 2002), the Wnt pathway that controls the EMS polarity involves WRM- $1 / \beta$-catenin and the POP-1/TCF transcription factor and hence is related to the canonical Wnt pathway. Unlike $\beta$-catenin in other organisms, WRM-1 does not bind to cadherins and functions in Wnt signaling only, but not in cell adhesion (Korswagen et al. 2000). In the canonical Wnt pathway, the Wnt signal regulates the stability and nuclear localization of $\beta$-catenin (Logan and Nusse 2004). However, it is not known how the Wnt signal regulates WRM-1, especially because WRM-1 does not have the conserved phosphorylation sites of GSK3 $\beta$. Furthermore, the subcellular localization of WRM-1 has not been determined. Therefore, the function of WRM-1 in the regulation of cell polarity is obscure.

In addition to the components of the Wnt pathway, LIT-1/MAP kinase and MOM-4/MAPKKK are involved in the EMS division (Meneghini et al. 1999; Rocheleau et al. 1999; Shin et al. 1999). MOM-4 activates the LIT-1 kinase, while LIT-1 binds to WRM-1 to phosphorylate POP-1. Activation of this Wnt/MAPK pathway results in the asymmetric distribution of POP-1 between the nuclei of the daughter cells (POP-1 asymmetry). Unlike the Numb and Prospero proteins in Drosophila, however, POP-1 does not localize to the cell cortex during division. Instead, POP-1 asymmetry is regulated by nuclear export (Lo et al. 2004). Although the nuclear export of POP-1 is regulated by phosphorylation by the LIT-1WRM-1 complex, it is not clear how the Wnt signaling pathway determines the difference in the rate of nuclear export between the daughter cells.

Here, we show that WRM-1 and LIT-1 localized asymmetrically to the anterior cell cortex before and during the division of post-embryonic cells. Surprisingly, after division, WRM-1 and LIT-1 localized preferentially to the nucleus of the posterior rather than anterior daughters. Our results suggest a role for cortical $\beta$-catenin in the regulation of cell polarity, and provide a novel link between cortical and nuclear $\beta$-catenin.

\section{Results and Discussion}

The Wnt pathway is involved in most asymmetric division during embryonic development in C. elegans (Kaletta et al. 1997; Lin et al. 1998). During post-embryonic development, the asymmetric division of some cells has been shown to require components of the Wnt pathway (Herman et al. 1995; Sawa et al. 1996; Rocheleau et al. 1999; Whangbo et al. 2000; Herman 2001; Siegfried and Kimble 2002). In particular, T-cell division is regulated by LIN-44/Wnt, LIN-17/Frizzled, LIT-1, and POP-1. However, no requirement for WRM-1 and MOM-4 in T-cell division has been demonstrated. To examine the requirement for wrm-1 in the asymmetric T-cell division, we analyzed a deletion mutant of wrm-1(tm514), 
which lacks part of the sixth exon. Using mosaic analyses, we found that $t m 514$ homozygous mutants were defective in their asymmetric T-cell division, as judged by the absence of phasmid socket cells (Supplementary Table S1). These defects were also observed in a wrm-1 temperaturesensitive (ts) mutant, ne1982. ne1982 appears to be hypomorphic even at the restrictive temperature, because ne1982 homozygotes from heterozygotes can grow to adults, while tm 514 homozygotes from heterozygotes die as embryos. We also found that a mom-4 ts mutant, ne1539, was defective in asymmetric T-cell division (Supplementary Table S1). These results indicate that wrm- 1 and mom- 4 are required for the asymmetric $\mathrm{T}$-cell division.

We also examined the effects of these ts mutations on the divisions of other cells, such as V1V6 and V5.p. Although we could not detect defects in the ts mom-4, lit-1 or wrm-1 single mutants, probably because these mutations are hypomorphic, the mom-4(ne1539); lit-1(t1512) double mutants showed defects in many post-embryonic divisions. These mutants lacked all Vcell-derived seam cells $(n=12$, as judged by the expression of ajm-1::GFP) (Koppen et al. 2001) and most P-cell-derived hypodermal cells at the L2 stage (data not shown), suggesting that the asymmetry of the $\mathrm{V}$ - and P-cell divisions was disrupted. The mutants also showed very uncoordinated movement, probably due to defects in motoneurons derived from the P cells. At the L2 stage, the V5.p cell, which normally produces anterior neuroblast and posterior hypoblast daughters, produced only neural cells in the double mutants (Supplementary Table S1). These observations suggest that most asymmetric divisions are regulated by a similar mechanism during post-embryonic development.

To elucidate the functions of WRM-1 and LIT-1, we analyzed their subcellular localization during postembryonic development using the WRM-1::GFP and GFP::LIT-1 fusion genes, which can rescue the wrm-1 and lit-1 mutants, respectively (Supplementary Table S1; Rocheleau et al. 1999). In the progeny of many cells, we first found that the nuclear level of WRM-1::GFP and GFP::LIT-1 was higher in the posterior daughters than in the anterior ones; this observation included hypodermal cells ( $T, V$, and $P$ cells), neuroblasts (QL and $Q R)$, and mesodermal cells (Mdr and Mvr) (Fig. 1A,F; Supplementary Fig. S1A,B,E,F; data not shown). The asymmetric nuclear localization of LIT-1 is also reported in embryos (Lo et al. 2004). We often detected weak punctate fluorescence at the anterior side of some cells before their division, for both GFP::LIT-1 and WRM-1::GFP (white arrowheads in Supplementary Fig. S1C,D; data not shown). The asymmetric localization of GFP ::LIT-1 and WRM-1::GFP was more clearly observed in seam cells (Vn.p), especially in the V5.p cell at the end of the L1 stage, when the expression of these genes was higher (Fig. 1B,G; Supplementary Fig. S1G). Punctate fluorescence was clearly visible near the cell membrane in the anterior half of the cells. The asymmetric cortical localization was also observed during the mitosis of the V5.p cell (Fig. 1C,D,H,I). No asymmetry in the cytoplasmic localization of WRM-1 or LIT-1 was observed before or during cell division. At telophase, their localization near the anterior cortex and in the newly formed posterior nucleus was observed (Fig. 1D,I). Although WRM$1::$ GFP was nearly undetectable in the anterior nucleus after the V5.p division, it was clearly detected in the anterior nucleus at telophase (Fig. 1, cf. D and E), suggesting that the WRM-1 protein is exported out of the anterior nucleus during late telophase. These results indicate that WRM-1 and LIT-1 are asymmetrically localized to the anterior cortex before and during division and to the posterior nucleus after the division of many cells during post-embryonic development.

We analyzed the dynamics of the nuclear localization of these proteins in the V5.p cell by fluorescence recovery after photobleaching (FRAP) experiments. Animals were nearly immobilized using pharmacological reagents (tricaine and tetramisole) (Knobel et al. 1999) to enable us to perform live analysis. The GFP fluorescence in defined regions of the V5.p cell was eliminated by photobleaching, followed by live analyses. In control experiments, application of the laser on the wild-type V5.p cell during its division did not disrupt the asymmetry of its subsequent lineage $(n=15)$. We first applied photobleaching to the entire V5.p cell at prophase (when the nucleolus starts to disappear but the nuclear envelope is still present) and found the fluorescence of WRM-1::GFP and GFP::LIT-1 was nearly undetectable after the division (Fig. 2A; data not shown for GFP::LIT-1), indicating that residual and newly generated fluorescence can be ignored in these experiments. Next, at prophase, we applied photobleaching either to the whole cell except for the nuclei (Fig. 2B; data not shown for GFP::LIT-1) or to the nuclei (Fig. 2C; data not shown for GFP::LIT-1). In both cases, a posterior nuclear localization was clearly detected after the division, indicating that both nuclear and cytoplasmic WRM-1 and LIT-1 localized to the posterior nucleus. Similarly, this asymmetric nuclear localization of the proteins was observed after photobleaching 
the anterior half of the cell, including the cortex, at anaphase (Fig. 2D,F) and after photobleaching the posterior half of the cell at telophase (Fig. 2E,G). After photobleaching the posterior half of the cell at telophase for GFP::LIT-1, the fluorescence in the anterior nucleus nearly disappeared, with a concomitant increase of fluorescence in the posterior nucleus (Fig. 2G), indicating that LIT-1 in the anterior nucleus accumulated in the posterior nucleus. These results show that LIT-1 and WRM-1 anywhere in the cell localized preferentially to the posterior nucleus.

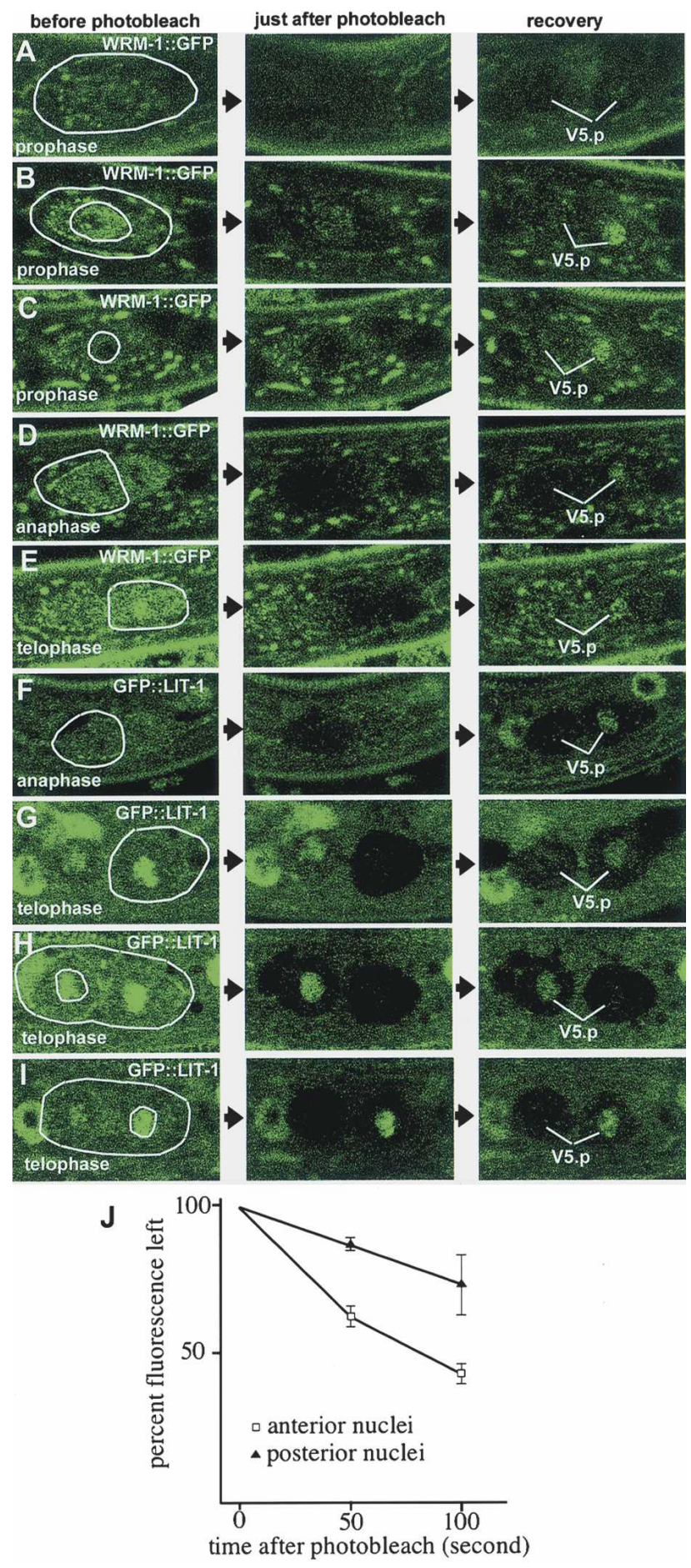

To analyze whether the asymmetry of the LIT-1 nuclear localization was produced by a difference in nuclear import or export rates, we applied photobleaching to eliminate fluorescence in the entire cell except either the anterior or posterior nucleus at telophase (Fig. $2 \mathrm{H}, \mathrm{I})$. After 50 and $100 \mathrm{sec}$, we measured how much fluorescence remained in the nucleus (Fig. 2J) and found that the posterior nucleus retained GFP::LIT-1 more efficiently than the anterior one. The results could be interpreted to indicate that the LIT-1 protein in the anterior nucleus was more unstable. However, this is unlikely, because the level of LIT-1 was not significantly decreased during the division (Fig. $1 \mathrm{H}-\mathrm{J}$ ). Furthermore, the above FRAP experiments showed that LIT-1 was exported out of the anterior nucleus and accumulated in the posterior nucleus (Fig. 2G). Therefore, the results suggest that the nuclear export rate of LIT-1 is higher in the anterior nucleus.

We analyzed the localization of WRM-1 and LIT-1 in the $\mathrm{T}$ and V5.p cells in mutants of the Wnt signaling pathway (Fig. 3; Table 1). In the following experiments, we used hypomorphic alleles for lit-1, mom-4, and wrm1. Therefore, results showing no effects of these mutations may not be conclusive. The T-cell division becomes symmetric in lin-17/frizzled mutants, while it is reversed (the fates of daughters are the reciprocal of what they are in wild type) in lin-44/wnt mutants. Consistent with this, the nuclear localization of WRM-1::GFP and GFP::LIT-1 was often reversed (higher in the anterior daughter) in lin-44 mutants (Fig. 3B,F). In lin-17 mutants, the nuclear localization of WRM-1::GFP and GFP ::LIT-1 was often very weak in both daughter cells (Fig. 3C,G). In lit-1 or mom-4 mutants, although WRM-1::GFP expression was still higher in the T.p cell, the expression was weak and uniform in the nucleus and cytoplasm (Fig. 3D; data not shown), suggesting that the MOM-4-LIT-1 pathway is required for the efficient nuclear localization of WRM-1. In mom-4 mutants, the difference in GFP::LIT-1 nuclear localization between daughter cells was much smaller than in wild type (Fig. $3 \mathrm{H})$, and sometimes the localization was symmetric (Table 1). The nuclear localization of GFP::LIT-1 was only weakly affected in the hypomorphic wrm-1 mutants (Table 1), most likely because the defect in the T-cell division was suppressed by GFP::LIT-1 (Supplementary Table S1), due to overexpression of the protein.

Figure 2. Dynamics of the localization examined by FRAP. $(A-I)$. Confocal images of the V5.p cell. Anterior is to the left; ventral is to the bottom. Each panel represents the same sample over time. The pictures on the left of each panel are the images before photobleaching. The bleached regions are circled. In $B, H$, and $I$, the laser was applied inside the large circle, but outside the small one. The GFPfusion genes used and stages in the cell cycle are indicated in each panel. The pictures in the middle of each panel are the images just after photobleaching, while those on the right show the recovery after bleaching. The pictures on the right of panels $A-G$ were recorded just after the completion of division, while those on the right of $H$ and $I$ were recorded $100 \mathrm{sec}$ after photobleaching. (J) The nuclear export rates of proteins from the anterior (squares) or posterior (triangles) nuclei were determined from the FRAP data shown in $H$ or $I$, respectively. The mean intensity of the fluorescence was calculated by the LSM510 software. The percentages of the fluorescence 50 or $100 \mathrm{sec}$ after photobleaching compared with those just after photobleaching are indicated on the $Y$-axis. Values are the means \pm SD of three (anterior nuclei) and four (posterior nuclei) independent experiments. 

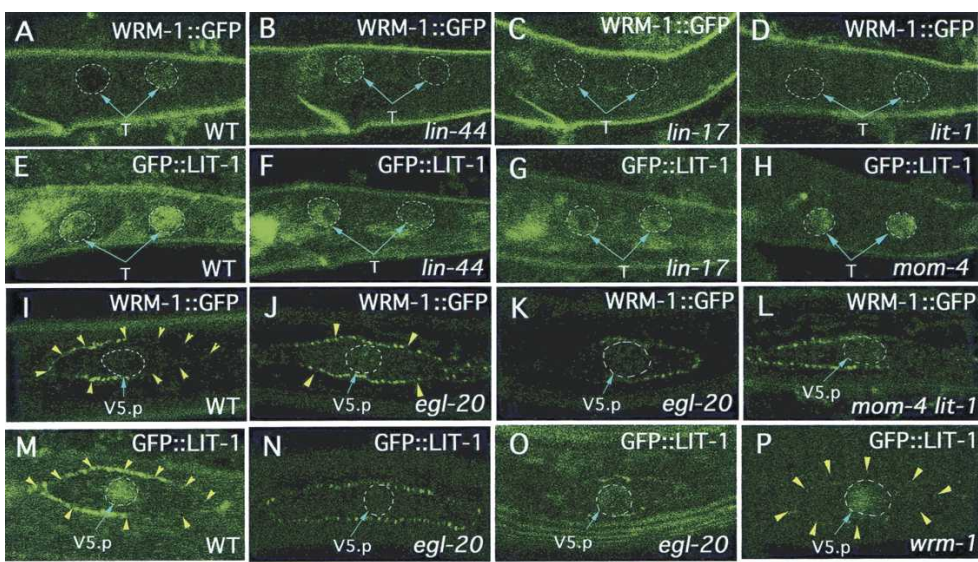

$\mathrm{H}$

GFP::LIT-1
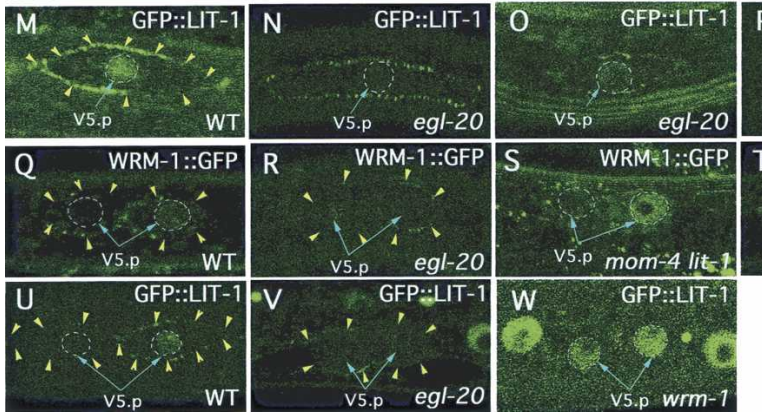

Figure 3. Genetic regulation of WRM-1::GFP and GFP ::LIT-1 localization. Anterior is to the left; ventral is to the bottom. Cell boundaries are indicated by yellow arrowheads where they are visible. Positions of nuclei are indicated by blue arrows, and shapes of the nuclei are outlined by dotted lines except in $R$ and $V$, in which the nucleus were not clearly detected because the cells were in telophase. The cells in the other panels are at interphase. $A-H$ are the T-cell daughters. $I-P$ show the V5.p cell. Q-W are the V5.p daughters. Genotypes are indicated in each panel. (WT) Wild type. The alleles were lin-44(n1792), lin-17(n3091), lit-1(t1512), mom-4(ne1539), egl-20(n585), and wrm-1(ne1982).

This indicates that the weak activity of mutated WRM-1 proteins could be compensated for by a high level of LIT1 , suggesting that the activity of WRM-1 may be regulated by LIT-1. Similarly, WRM-1::GFP suppressed the T-cell defects of lit-1 and mom-4 hypomorphic mutants (Supplementary Table S1). This is consistent with the previous observation that the kinase activity of LIT-1 is regulated by WRM-1 (Rocheleau et al. 1999).

Because the cortical localization of WRM-1 and LIT-1 was detected at only a low frequency in the $\mathrm{T}$ cell, we analyzed it in the V5.p cell. The division of V5.p is regulated by egl-20/wnt (Whangbo et al. 2000). In egl-20 mutants, this division is often reversed $32 \%$ reversed, $68 \%$ normal $n=78$ ); that is, the polarity of the division is nearly random. Consistent with this phenotype, the cortical localization of WRM-1 and LIT-1 was randomized in the mutants, where it was symmetric or enriched anteriorly or posteriorly (Fig. 3J,K,N,O; Table 2). In contrast to the observation before division, during division of the V5.p cell, symmetric cortical localization of WRM-1 and LIT-1 was very rarely observed (Table 2). This indicates that in egl20 mutants, the initial symmetric cortical localization became asymmetric (either anterior or posterior enriched) before the start of division. After division, the nuclear localization was always asymmetric (higher either in the anterior or posterior nucleus). Despite the nearly random asymmetry, at telophase the localization of these molecules in the cortex was always the opposite of that in the nucleus $(n=5$ for WRM-1::GFP and $n=1$ for GFP::LIT-1) (Fig. 3R,V). We also analyzed the WRM-1::GFP localization in live immobilized egl-20 animals. In all five animals examined, the localization of WRM-1 in the cortex during division was the opposite of that in the nucleus after division, indicating a tight and reverse correlation between the cortical and nuclear asymmetry. The wrm-1(ne1982) mutation did not affect the V5.p division in terms of lineage (Supplementary Table S1). However, the cortical GFP::LIT-1 localization was clearly reduced and detected only with a higher laser power than we had used in our other experiments (Fig. 3P). After division, differences in the nuclear GFP::LIT-1 level between daughter cells were significantly smaller in the wrm-1 mutants (Fig. 3W) than in wild type. These results indicate that WRM-1 was required for the cortical localization and the asymmetric nuclear localization of LIT-1. The mom-4(ne1539) and lit-1(t1512) single mutations did not affect the cortical and nuclear localization of WRM-1::GFP in the V5.p division (data not shown), consistent with the observation that they did not affect the cell fates in this lineage. In the mom-4; lit-1 double mutants, the nuclear localization after division was significantly affected, as was the case for T-cell division in mom-4 or lit-1 single mutants. In most cases (16 of 22 animals), localization was still asymmetric, but cytoplasmic fluorescence in the V5.pp cell was significantly increased (Fig. 3, cf. Q and S). In some animals (four of 22 animals), localization was nearly symmetric and uniform in both cytoplasm and nucleus (Fig. 3T). Despite the defects in nuclear localization, the cortical localization of WRM-1::GFP was not significantly affected (Fig. 3L). These results indicate that the MOM-4LIT-1 pathway is required for the nuclear but probably not for the cortical localization of WRM-1. Taken to-

Table 1. Genetic regulation of WRM-1 and LIT-1 nuclear localization in T-cell division

\begin{tabular}{|c|c|c|c|c|c|c|c|c|c|}
\hline \multirow[b]{2}{*}{ Genotype } & \multicolumn{5}{|c|}{ WRM-1::GFP } & \multicolumn{4}{|c|}{ GFP ::LIT-1 } \\
\hline & T.a $>$ T.p & $\mathrm{T} \cdot \mathrm{a}=\mathrm{T} \cdot \mathrm{p}$ & T.a $<$ T.p & UD & $n$ & T.a > T.p & $\mathrm{T} \cdot \mathrm{a}=\mathrm{T} \cdot \mathrm{p}$ & T.a $<$ T.p & $n$ \\
\hline $\mathrm{N} 2$ & $0 \%$ & $0 \%$ & $65 \%$ & $35 \%$ & 52 & $2 \%$ & $2 \%$ & $96 \%$ & $\overline{46}$ \\
\hline lin-44(n1792) & $44 \%$ & $0 \%$ & $21 \%$ & $35 \%$ & 34 & $72 \%$ & $14 \%$ & $14 \%$ & 21 \\
\hline $\operatorname{lin}-17(n 3091)$ & $5 \%$ & $27 \%$ & $19 \%$ & $49 \%$ & 41 & $15 \%$ & $81 \%$ & $4 \%$ & 27 \\
\hline wrm-1(ne1982) & NA & NA & NA & NA & - & $0 \%$ & $8 \%$ & $92 \%$ & 62 \\
\hline mom-4(ne1539) & $0 \%$ & $0 \%$ & $77 \%$ & $23 \%$ & 39 & $3 \%$ & $25 \%$ & $72 \%$ & 32 \\
\hline lit-1(t1512) & $0 \%$ & $0 \%$ & $60 \%$ & $40 \%$ & 43 & NA & NA & NA & - \\
\hline
\end{tabular}

(UD) undetectable; (NA) not applicable. 
Table 2. Localization of WRM-1 and LIT-1 in the V5.p division in egl-20 mutants

\begin{tabular}{lrrr}
\hline & $\mathrm{A}>\mathrm{P}$ & $\mathrm{A}=\mathrm{P}$ & $\mathrm{A}<\mathrm{P}$ \\
\hline Cortical WRM-1::GFP before division & 22 & 14 & 6 \\
Cortical WRM-1 ::GFP during division & 9 & 0 & 9 \\
Nuclear WRM-1::GFP after division & 11 & 0 & 16 \\
Cortical GFP $:$ LIT-1 before division & 8 & 11 & 8 \\
Cortical GFP $:$ LIT-1 during division & 4 & 1 & 2 \\
Nuclear GFP ::LIT-1 after division & 10 & 0 & 21 \\
\hline
\end{tabular}

(A) anterior side; $(\mathrm{P})$ posterior side. Numbers of animals are indicated.

gether, our results show that Wnt signals regulate the asymmetric cortical and nuclear localization of LIT-1 and WRM-1.

We have shown a perfect correlation between the WRM-1 cortical and nuclear asymmetry in egl-20 mutants, in which the orientation of polarity is nearly random. In addition, in wrm-1 mutants, in which the cortical LIT-1 localization was weak, there was also little nuclear LIT-1 asymmetry. These results suggest that the cortical and nuclear asymmetries are regulated by the same mechanism or that the cortical asymmetry regulates the nuclear one. EGL-20, which regulates the V5.p cell division, is expressed at the posterior side of the V5.p cell (Whangbo and Kenyon 1999). In egl-20 mutants, we often observed the symmetric localization of WRM-1 before the division. Therefore, EGL-20 appears to function to remove cortical WRM-1 from the posterior cortex, probably by releasing it into cytoplasm. One possibility is that the released WRM-1 stays in the posterior cytoplasm and is imported into the posterior nucleus at telophase. However, the FRAP experiments showed that, even if the proteins were in the anterior cytoplasm or nucleus before or during division, they preferentially localized to the posterior nucleus after division. In addition, we did not observe cytoplasmic asymmetry of their localization. Therefore, it is likely that WRM-1 released from the posterior cortex diffuses throughout the cytoplasm. In this case, the released molecules do not carry the polarity information that is essential to accomplish the asymmetric nuclear localization. Therefore, the cortical asymmetry generated by the Wnt signals may function to establish and to maintain the polarity. In this model, Wnts have two distinct functions: to activate WRM-1 by releasing it from the cortex and to establish polarity by generating the cortical asymmetry. At telophase, the cortical asymmetry might regulate the nuclear asymmetry. We found that at telophase the daughters had different nuclear export rates for LIT-1. In addition, WRM-1 that binds to LIT-1 was observed in the anterior nucleus at early telophase, and it disappeared at late telophase. Therefore, the polarity is likely to regulate the nuclear export or retention of the LIT-1-WRM-1 complex. In contrast to the posterior nuclear localization of these proteins, the nuclear level of POP-1 is higher in anterior daughters (Lin et al. 1995; Herman 2001). In addition, we previously showed that the nuclear localization of PSA-1/SWI3 and PSA-4/SWI2 is similar between the T-cell daughters at telophase (Sawa et al. 2000). Therefore, the difference in nuclear export rates was not due to differences in the activity of the general export machinery.
How is the cortical WRM-1 and LIT-1 asymmetry established by Wnt? It has been recently reported that ectopically expressed MOM-5/Frizzled localizes to the posterior cortex of some post-embryonic cells, including the $\mathrm{T}$ cell (Park et al. 2004). Although it is not known whether MOM-5 is normally expressed in these cells, and mom-5 mutants are not defective in T-cell division (H. Sawa, unpubl.), these cells at least have the ability to localize Frizzled receptors. Therefore, it is tempting to speculate that LIN-17/Frizzled is localized to the posterior cortex of the $\mathrm{T}$ cell to regulate an asymmetric release of WRM-1 and LIT-1 from the cortex.

It has been reported that LIT-1 regulates the nuclear export of POP-1 (Lo et al. 2004). Our results indicate that LIT-1 also functions in the nuclear localization of WRM1. It has also been reported that POP-1 functions in the daughter cells with a higher POP-1 concentration in embryos (Lin et al. 1995, 1998), while in post-embryonic development POP-1 functions in daughters with a lower POP-1 level (Herman 2001; Siegfried et al. 2004). Therefore, the POP-1 asymmetry does not explain its roles in post-embryonic development. In addition, a form of POP-1 that does not have the putative $\beta$-catenin-binding domain can rescue pop-1 mutants in the embryo (Maduro et al. 2002), while its overexpression in larvae causes the same T-cell division defect that is seen with pop-1 mutants (Herman 2002). Therefore, pop-1 functions as a transcriptional repressor in the embryo, as reported (Calvo et al. 2001), while it appears to act as an activator in post-embryonic development (Herman 2001). Our results suggest that the nuclear WRM-1 together with POP-1 in posterior daughters functions as a transcriptional activator to regulate asymmetric cell fates in post-embryonic cells.

Here we propose that Wnt signals regulate cell polarity through the asymmetric cortical localization of $\beta$-catenin in $C$. elegans. Asymmetric $\beta$-catenin localization is observed in many cell types, such as hematopoietic stem cells, that presumably undergo asymmetric divisions (Zhang et al. 2003). Although cortical $\beta$-catenin is believed to function in cell adhesion, our results suggest a possibility that it is involved in Wnt signaling and/or the regulation of cell polarity.

\section{Materials and methods}

\section{Genetics}

wrm-1(tm514) was isolated by the National BioResource Project (http:// www.nbrp.jp/index.jsp). wrm-1(ne1982) and mom-4(ne1539) were kindly provided by K. Nakamura and C. Mello (University of Massachusetts, Worcester, MA). The tm514 homozygotes from heterozygous mothers die as embryos. We generated $t m 514$ homozygotes that carry a transgene containing the wrm-1 genomic DNA (C29B1) and sur-5::GFP as a cellautonomous marker. We then identified mosaic animals in which the $\mathrm{T}$ cells did not have the transgene. After recovery of the mosaic animals, we analyzed the phenotype at the $\mathrm{L} 2$ stage. To analyze temperature-sensitive mutants of wrm-1, lit-1, and mom-4, animals grown at $15^{\circ} \mathrm{C}$ were shifted to $25^{\circ} \mathrm{C}$ at the late embryonic stage. mom-4; lit-1 double mutants that were lethal at $15^{\circ} \mathrm{C}$ were grown at $11.5^{\circ} \mathrm{C}$ and then shifted to $25^{\circ} \mathrm{C}$ at the late-embryonic stage or at the middle L1 stage only for analyses of theV5.p division. Other strains were grown at $22.5^{\circ} \mathrm{C}$.

Analyses of WRM-1 and LIT-1 localization

The WRM-1::GFP construct (pWRM4) contains a 5.9-kb BstZ17I-BlpI fragment of C29B1 and its PCR fragment (from the BlpI site to the end of the wrm-1-coding sequence) ligated to the Venus version (Nagai et al. 2002) of the GFP gene (a gift from Dr. T. Ishihara, Kyusyu University, 
Fukuoka, Japan). The construct was transformed to the unc-76(e911) strain with a unc-76 rescuing plasmid (Bloom and Horvitz 1997) to obtain an extrachromosomal array osEx158. All the strains with osEx158 also carried unc-76(e911). GFP::LIT-1 was described previously (Rocheleau et al. 1999|. Analyses of GFP localization and FRAP experiments were performed using a confocal microscopy (LSM510 Zeiss). For FRAP, fluorescence in defined regions was bleached out by applying a laser at maximum power nine to 12 times while manually shifting focal planes.

\section{Acknowledgments}

We are grateful to K. Nakamura and C. Mello for wrm-1 and mom-4 mutants, F. Matsuzaki and members of Sawa lab for comments on the manuscript, Takeshi Ishihara for the Venus plasmid, and C. elegans Genetic Center and National Bioresource Project for strains. This work was supported by grants from the Japanese Ministry of Education, Culture, Sports, Science and Technology and the Japan Society for the Promotion of Science (H.S.).

\section{References}

Adler, P.N. 2002. Planar signaling and morphogenesis in Drosophila. Dev. Cell 2: 525-535.

Betschinger, J. and Knoblich, J.A. 2004. Dare to be different: Asymmetric cell division in Drosophila, C. elegans and vertebrates. Curr. Biol. 14: R674-R685.

Bloom, L. and Horvitz, H.R. 1997. The Caenorhabditis elegans gene unc-76 and its human homologs define a new gene family involved in axonal outgrowth and fasciculation. Proc. Natl. Acad. Sci. 94: 34143419.

Calvo, D., Victor, M., Gay, F., Sui, G., Luke, M.P., Dufourcq, P., Wen, G., Maduro, M., Rothman, J., and Shi, Y. 2001. A POP-1 repressor complex restricts inappropriate cell type-specific gene transcription during Caenorhabditis elegans embryogenesis. EMBO J. 20: 7197-7208.

Herman, M. 2001. C. elegans POP-1/TCF functions in a canonical Wnt pathway that controls cell migration and in a noncanonical Wnt pathway that controls cell polarity. Development 128: 581-590.

- 2002. Control of cell polarity by noncanonical Wnt signaling in C. elegans. Semin. Cell Dev. Biol. 13: 233-241.

Herman, M.A., Vassilieva, L.L., Horvitz, H.R., Shaw, J.E., and Herman, R.K. 1995. The C. elegans gene lin-44, which controls the polarity of certain asymmetric cell divisions, encodes a Wnt protein and acts cell nonautonomously. Cell 83: 101-110.

Horvitz, H.R. and Herskowitz, I. 1992. Mechanisms of asymmetric cell division: Two Bs or not two Bs, that is the question. Cell 68: 237-255.

Kaletta, T., Schnabel, H., and Schnabel, R. 1997. Binary specification of the embryonic lineage in Caenorhabditis elegans. Nature 390: 294298.

Kemphues, K. 2000. PARsing embryonic polarity. Cell 101: 345-348.

Knobel, K.M., Jorgensen, E.M., and Bastiani, M.J. 1999. Growth cones stall and collapse during axon outgrowth in Caenorhabditis elegans. Development 126: 4489-4498.

Koppen, M., Simske, J.S., Sims, P.A., Firestein, B.L., Hall, D.H., Radice, A.D., Rongo, C., and Hardin, J.D. 2001. Cooperative regulation of AJM-1 controls junctional integrity in Caenorhabditis elegans epithelia. Nat. Cell Biol. 3: 983-991.

Korswagen, H.C. 2002. Canonical and non-canonical Wnt signaling pathways in Caenorhabditis elegans: Variations on a common signaling theme. Bioessays 24: 801-810.

Korswagen, H.C., Herman, M.A., and Clevers, H.C. 2000. Distinct $\beta$-catenins mediate adhesion and signalling functions in C. elegans. Nature 406: 527-532.

Lin, R., Thompson, S., and Priess, J.R. 1995. pop-1 encodes an HMG box protein required for the specification of a mesoderm precursor in early C. elegans embryos. Cell 83: 599-609.

Lin, R., Hill, R.J., and Priess, J.R. 1998. POP-1 and anterior-posterior fate decisions in C. elegans embryos. Cell 92: 229-239.

Lo, M.C., Gay, F., Odom, R., Shi, Y., and Lin, R. 2004. Phosphorylation by the $\beta$-catenin/MAPK complex promotes 14-3-3-mediated nuclear export of TCF/POP-1 in signal-responsive cells in C. elegans. Cell 117: 95-106.

Logan, C.Y. and Nusse, R. 2004. The Wnt signaling pathway in development and disease. Annu. Rev. Cell. Dev. Biol. 20: 781-810.
Maduro, M.F., Lin, R., and Rothman, J.H. 2002. Dynamics of a developmental switch: Recursive intracellular and intranuclear redistribution of Caenorhabditis elegans POP-1 parallels Wnt-inhibited transcriptional repression. Dev. Biol. 248: 128-142.

Meneghini, M.D., Ishitani, T., Carter, J.C., Hisamoto, N., NinomiyaTsuji, J., Thorpe, C.J., Hamill, D.R., Matsumoto, K., and Bowerman, B. 1999. MAP kinase and Wnt pathways converge to downregulate an HMG-domain repressor in Caenorhabditis elegans. Nature 399: 793797.

Nagai, T., Ibata, K., Park, E.S., Kubota, M., Mikoshiba, K., and Miyawaki, A. 2002. A variant of yellow fluorescent protein with fast and efficient maturation for cell-biological applications. Nat. Biotechnol. 20: $87-90$.

Park, F.D., Tenlen, J.R., and Priess, J.R. 2004. C. elegans MOM-5/frizzled functions in MOM-2/Wnt-independent cell polarity and is localized asymmetrically prior to cell division. Curr. Biol. 14: 2252-2258.

Rocheleau, C.E., Yasuda, J., Shin, T.H., Lin, R., Sawa, H., Okano, H., Priess, J.R., Davis, R.J., and Mello, C.C. 1999. WRM-1 activates the LIT-1 protein kinase to transduce anterior/posterior polarity signals in C. elegans. Cell 97: 717-726.

Roegiers, F. and Jan, Y.N. 2004. Asymmetric cell division. Curr. Opin. Cell. Biol. 16: 195-205.

Sawa, H., Lobel, L., and Horvitz, H.R. 1996. The Caenorhabditis elegans gene lin-17, which is required for certain asymmetric cell divisions, encodes a putative seven-transmembrane protein similar to the Drosophila frizzled protein. Genes \& Dev. 10: 2189-2197.

Sawa, H., Kouike, H., and Okano, H. 2000. Components of the SWI/SNF complex are required for asymmetric cell division in C. elegans. Mol. Cell 6: 617-624.

Shin, T.H., Yasuda, J., Rocheleau, C.E., Lin, R., Soto, M., Bei, Y., Davis, R.J., and Mello, C.C. 1999. MOM-4, a MAP kinase kinase kinaserelated protein, activates WRM-1/LIT-1 kinase to transduce anterior/ posterior polarity signals in C. elegans. Mol. Cell 4: 275-280.

Siegfried, K.R. and Kimble, J. 2002. POP-1 controls axis formation during early gonadogenesis in C. elegans. Development 129: 443-453.

Siegfried, K.R., Kidd, A.R., Chesney, M.A., and Kimble, J. 2004. The sys-1 and sys-3 genes cooperate with Wnt signaling to establish the proximal-distal axis of the Caenorhabditis elegans gonad. Genetics 166: $171-186$.

Thorpe, C.J., Schlesinger, A., and Bowerman, B. 2000. Wnt signalling in Caenorhabditis elegans: Regulating repressors and polarizing the cytoskeleton. Trends Cell Biol. 10: 10-17.

Whangbo, J. and Kenyon, C. 1999. A Wnt signaling system that specifies two patterns of cell migration in C. elegans. Mol. Cell 4: 851-858.

Whangbo, J., Harris, J., and Kenyon, C. 2000. Multiple levels of regulation specify the polarity of an asymmetric cell division in C. elegans. Development 127: 4587-4598.

Zhang, J., Niu, C., Ye, L., Huang, H., He, X., Tong, W.G., Ross, J., Haug, J., Johnson, T., Feng, J.Q., et al. 2003. Identification of the haematopoietic stem cell niche and control of the niche size. Nature 425: 836-841. 


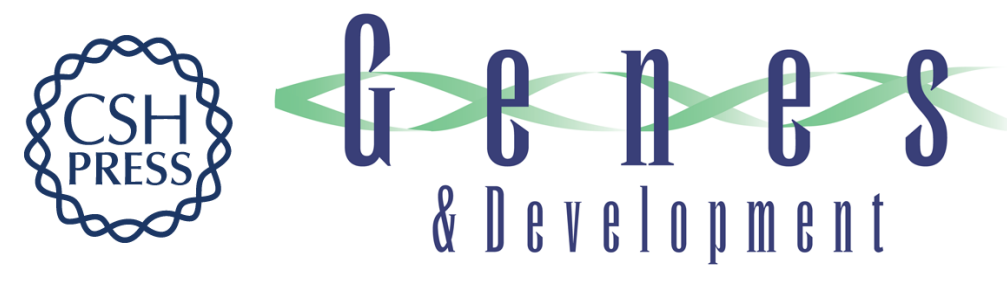

\section{Asymmetric cortical and nuclear localizations of WRM-1/ $\beta$-catenin during asymmetric cell division in C. elegans}

Hisako Takeshita and Hitoshi Sawa

Genes Dev. 2005, 19:

Access the most recent version at doi:10.1101/gad.1322805

Supplemental http://genesdev.cshlp.org/content/suppl/2005/07/29/19.15.1743.DC1
Material

References This article cites 32 articles, 7 of which can be accessed free at:

http://genesdev.cshlp.org/content/19/15/1743.full.html\#ref-list-1

License

Email Alerting Receive free email alerts when new articles cite this article - sign up in the box at the top

Service right corner of the article or click here.

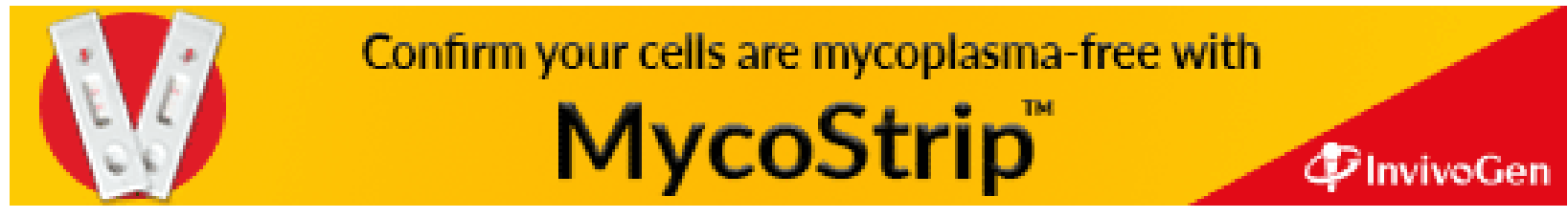

